\title{
FUNCIONAMIENTO EJECUTIVO EN ESTUDIANTES CON DIFERENTES NIVELES DE COMPRENSIÓN LECTORA
}

\author{
Rebeca Siegenthaler Hierro \\ (siegenth@uji.es) \\ Javier Rello Segovia \\ Jessica Mercader Ruiz \\ Ma Jesús Presentación Herrero \\ Universidad Jaume I de Castellón
}

Fecha de Recepción: 8 Marzo 2018

Fecha de Admisión: 10 Abril 2018

\section{RESUMEN}

El objetivo del presente estudio consistió en examinar las diferencias en el funcionamiento ejecutivo (FE) entre dos grupos de sujetos de último ciclo de Educación Primaria con distintos niveles de comprensión lectora (medio-alto y medio-bajo). La muestra del estudio estuvo conformada por 148 niños (78 y 70 respectivamente). A los participantes se les administró tareas neuropsicológicas relacionadas con las funciones ejecutivas de memoria de trabajo, inhibición y atención. Para evaluar el FE conductual, sus profesores cumplimentaron también el cuestionario Behavior Rating Inventory of Executive Function (BRIEF, Gioia, Isquith, Guy y Kenworthy, 2000). Los resultados evidenciaron diferencias entre ambos grupos en tareas neuropsicológicas de memoria de trabajo y atención. Especialmente importantes fueron las diferencias encontradas en todas las variables incluidas en el índice de metacognición del BRIEF cumplimentado por los profesores. Se comentan las implicaciones de estos hallazgos para la investigación y la práctica psicoeducativa.

Palabras clave: funcionamiento ejecutivo; comprensión lectora; memoria de trabajo; atención; metacognición

\section{ABSTRACT}

Executive functioning in students with different levels of reading comprehension.

The aim of this study was to analyze the differences in executive functioning (EF) between two groups of subjects in the last cycle of Primary Education with different levels of reading comprehension (medium-high and medium-low). The sample consisted of 148 children (78 and 70 respectively). The participants passed neuropsychological tasks related to the executive functions of working memory, inhibition and attention. To evaluate the behavioral FE, their teachers also completed the questionnaire Behavior Rating Inventory of Executive Function (BRIEF, Gioia, Isquith, Guy and 


\section{FUNCIONAMIENTO EJECUTIVO EN ESTUDIANTES CON DIFERENTES NIVELES DE COMPRENSIÓN LECTORA}

Kenworthy, 2000). The results showed differences between both groups in neuropsychological tasks of working memory and attention. Especially important were the differences found in all the factors included in the metacognition index of the BRIEF completed by the teachers. The implications of these findings for research and psychoeducational practice are discussed.

Key words: executive functioning; reading comprehension; working memory; attention; metacognition

\section{ANTECEDENTES DE LA TEMÁTICA A TRATAR}

Los informes internacionales (PISA, PIRLS, EECL o PIAC) ponen de manifiesto que los problemas de comprensión lectora, no sólo en niños sino en adolescentes y adultos, son importantes. En España concretamente, el informe PISA (2015) reporta que los problemas de comprensión lectora se presentan en un $25 \%$ de los alumnos.

La comprensión lectora ha sido objeto de estudio desde hace décadas ya que la importancia que tiene en el desarrollo integral de la persona es indiscutible. Las dificultades de lectura en general y de comprensión lectora en particular pueden tener consecuencias negativas en la vida de las personas ya que son fundamentales para muchos aspectos de la misma. Son varios los factores que parecen estar implicados en las dificultades de comprensión lectora. Destaca el papel de la inteligencia (Spinillo, 2013), la comprensión oral (Blázquez-Garcés et al., 2015), Ios procesos básicos de lectura (Cuetos, Rodríguez, Ruano y Arribas, 2007), las variables afectivo-motivacionales (Mezzalira y Boruchovitch, 2014) así como diferentes factores cognitivos (Filippetti y López, 2014). En relación con estos últimos, son muchos los estudios que asocian el funcionamiento ejecutivo con la decodificación o la velocidad lectora, sin embargo, son menos los que estudian su relación con la comprensión (Filippetti y López, 2016). Este hecho, unido a la importancia crucial de los factores cognitivos, resalta la necesidad de seguir investigando con el fin de comprender el origen de esta problemática.

La memoria de trabajo es la función ejecutiva más analizada en relación con la comprensión lectora, habiéndose comprobado su relación tanto en muestras de lectores expertos (Swanson y Alexander, 1997), con dificultades lectoras (Sesma, Mahone, Levine, Eason y Cutting, 2009) y en malos comprendedores (Canet-Juric, Burin, Andrés y Urquijo, 2013). Los sujetos con un rendimiento alto en las medidas de memoria operativa tienden a emplear buenas estrategias de comprensión lectora mientras que los estudiantes que obtienen puntuaciones bajas suelen rendir por debajo de la media (Cain y Oakhill, 2004; Carretti et al., 2009; García-Madruga y Fernández, 2008; Gómez Veiga, Vila, García- Madruga, Contreras y Elosúa, 2013). López-Escribano, De Juan, GómezVeiga y García-Madruga (2013) concluyen que la memoria de trabajo influye en la comprensión lectora, hasta el punto de que la afectación de una conlleva a la afectación de la otra.

En relación con la atención, diferentes trabajos informan que la atención y la comprensión lectora se relacionan de forma directa (Kendeou, Van den Broek, Helder y Karlsson, 2014; Miranda, Fernández, Robledo y García, 2010). El déficit de atención perjudica la monitorización del proceso de comprensión del texto (McInnes, Humphries, Hogg- Johnson y Tannock, 2003). Así mismo, los sujetos con inatención son más susceptibles de distraerse con información irrelevante, sobre todo si el texto es largo (Solan, Shelley-Trembaly, Hansen y Larson, 2007). Una investigación con estudiantes con y sin TDAH de cuarto, quinto y sexto de Educación Primaria (EP) evidenció una menor competencia para extraer ideas principales de los textos en los alumnos con dificultades atencionales (Brock y Knapp, 1996). También Ghelani, Sidhu, Jain y Tannock (2004) comprobaron que estudiantes adolescentes sin TDAH obtenían mejor resultado que los alumnos con TDAH en la comprensión de textos cortos. 
Por otro lado, los procesos de inhibición también parecen estar relacionados con la comprensión lectora (Kendeou et al., 2014). Se ha comprobado que unos mecanismos ineficientes de inhibición pueden llevar a un mal rendimiento en comprensión lectora (Carretti et al., 2009; Kendeou et al., 2014). Los niños con dificultades de comprensión lectora muestran problemas a la hora de suprimir 0 eliminar la información irrelevante (Cain, 2006). Este autor ha comprobado que cuando se les dice a los niños que ignoren palabras irrelevantes de la lectura, los que tienen problemas de inhibición no lo hacen, y recuerdan las palabras irrelevantes en mayor medida que los niños sin estos problemas. Por otro lado, Cartoceti (2012) ha evidenciado la relación existente entre el control inhibitorio y la comprensión lectora. Este autor informa que los lectores con dificultades de comprensión presentan déficits en los mecanismos de inhibición y supresión de la información, principalmente de tipo verbal, lo que impide que puedan seleccionar las ideas más importantes.

Finalmente, son muy escasos los estudios que han analizado la relación de la comprensión lectora con el funcionamiento ejecutivo conductual. El cuestionario más utilizado para este fin en el ámbito de la investigación es el BRIEF, Behavior Rating Inventory of Executive Functions (Gioia, Isquith, Guy y Kenworthy, 2000). A través de este cuestionario, en su versión para padres, Prat (2000) encuentra dificultades en los alumnos con problemas lectores en memoria de trabajo y planificación. Más importantes son las diferencias encontradas por Gioia, Isquith, Kenworthy y Barton (2002) con una muestra de estudiantes con y sin dificultades de comprensión lectora de 8 a 15 años. Estos autores informan de mayor problematicidad en inhibición, memoria de trabajo, planificación/organización y monitoreo en el grupo con dificultades comparado con el grupo sin dificultades. En la misma línea, Locascio, Mahone, Eason, y Cutting (2010) obtienen también diferencias significativas entre el grupo control y el grupo con dificultades de comprensión en el índice de funcionamiento ejecutivo global.

La revisión realizada sobre las diferencias en el funcionamiento ejecutivo, tanto a nivel cognitivo como conductual, entre niños con diferentes niveles de comprensión lectora, pone de manifiesto la necesidad de continuar investigando, con la finalidad de determinar el papel de las mismas en el rendimiento lector. Son necesarios trabajos que identifiquen el peso de estas funciones con el fin de prevenir futuros problemas mediante intervenciones específicas para ello.

\section{OBJETIVO DE LA INVESTIGACIÓN}

El objetivo de este trabajo es analizar las diferencias en el funcionamiento ejecutivo (FE) entre dos grupos de sujetos de último ciclo de Educación Primaria con distintos niveles de comprensión lectora (medio-alto y medio-bajo). Concretamente, se analizan las FE de memoria de trabajo, inhibición y atención, así como las FE conductuales valoradas por los profesores.

\section{MUESTRA Y PARTICIPANTES}

En este trabajo han participado 148 estudiantes (55 niños y 93 niñas) de entre 10 y 12 años (Media $=10.70 \pm 0.67$ ) y las 7 tutoras de los mismos. Ninguno de los sujetos de la muestra presentaba discapacidad intelectual (media $\mathrm{Cl}=111.46 \pm 12.04$ ), ni trastornos del neurodesarrollo, deficiencias sensoriales, problemas psicológicos graves o deprivación sociocultural. Un $62.8 \%$ de los participantes cursaba $5^{\circ}$ y un $37.2 \%$ cursaba 6을 de Educación Primaria. En relación con los datos sociodemográficos, la mayoría de la muestra era de nacionalidad española $(98.7 \%)$.

Para la realización de este estudio se dividió la muestra en dos grupos en función del nivel de comprensión lectora, medio-bajo y medio-alto. El primer grupo lo formaban los alumnos (n $=70$ ) con nivel medio-bajo en comprensión lectora (percentil < 50). El segundo grupo estuvo formado por los alumnos ( $n=78$ ) con nivel medio-alto en comprensión lectora (percentil $\geq 50$ ). 


\section{FUNCIONAMIENTO EJECUTIVO EN ESTUDIANTES CON DIFERENTES NIVELES DE COMPRENSIÓN LECTORA}

\section{METODOLOGíA}

\section{Instrumentos utilizados}

Evaluación neuropsicológica del funcionamiento ejecutivo

Se han evaluado distintos componentes neuropsicológicos de funcionamiento ejecutivo. Concretamente la memoria de trabajo, la atención y la inhibición. Para evaluar la memoria de trabajo se han utilizado dos subpruebas (Test de Dígitos y Test de Letras y Números) de la IV Escala de Inteligencia de Wechsler para niños (WISC-IV; Wechsler, 2010) y para medir la atención y la inhibición el test D2 (Seisdedos, 2012).

Test de Dígitos (WISC-IV; Wechsler, 2010). Esta subprueba consta de dos tareas de aplicación independiente: "Dígitos en orden directo" y "Dígitos de orden inverso". Hay un total de 8 elementos en cada tarea, con dos intentos en cada uno.

Test de Letras y Números (WISC-IV; Wechsler, 2010). En esta prueba se lee al niño una combinación de letras y números y su tarea consiste en repetir la secuencia, primero los números en orden ascendente y luego las letras. Hay 10 elementos con tres intentos para cada elemento.

Test d2, Test de Atención (Seisdedos, 2012). La prueba consiste en marcar en un grupo de letras aquellas que son una "d" y tienen dos rayas arriba, dos rayas abajo 0 una raya arriba y otra abajo. Consta de 14 líneas de letras y el alumno tiene 20 segundos para cada línea. Se tiene en cuenta el total de comisiones y de omisiones.

\section{Evaluación conductual del funcionamiento ejecutivo}

Para realizar la evaluación del FE conductual, se ha utilizado la Behavior Rating Inventory of Executive Function para profesores (BRIEF; Gioia, Isquith, Guy y Kenworthy, 2000). Se trata de un cuestionario que mide el funcionamiento ejecutivo de niños y adolescentes (5-18 años) a través de la observación conductual de los profesores. Consta de 86 ítems que se puntúan a través de una escala tipo Likert ( 1 = nunca, 2 = a veces, $3=$ frecuentemente) agrupados en 8 escalas: inhibición, cambio, control emocional, iniciativa, memoria de trabajo, planificación/organización, organización de materiales y monitoreo. Estas escalas se agrupan en dos índices, el de regulación comportamental y el de metacognición. Las tres primeras forman el índice de regulación comportamental y el resto el de metacognición. El índice de regulación comportamental evalúa la capacidad del niño para cambiar de estado afectivo y modular las emociones y la conducta mediante un apropiado autocontrol. El índice de metacognición analiza la capacidad del niño para iniciar, planificar, organizar y mantener en la memoria operativa la resolución de problemas. La prueba ofrece también una puntuación global de los déficits de funcionamiento ejecutivo.

\section{Procedimiento}

Para la realización de esta investigación se obtuvieron los permisos de la Consellería de Educación de la Generalitat Valenciana, la aprobación del Comité Ético de la Universidad Jaume I, así como el consentimiento informado de los padres. El estudio se realizó con estudiantes de un Centro Educativo de Castellón durante dos años consecutivos. El primer año se seleccionó como muestra los grupos de alumnos de $5^{0}$ y $6^{0}$ de EP. El segundo año sólo participaron los sujetos de $5^{0}$ de EP. El pase de las pruebas se realizó durante los dos cursos escolares en el periodo comprendido entre diciembre y marzo. Dichas pruebas fueron aplicadas por evaluadores cualificados en espacios preparados adecuadamente para tal fin. Las pruebas de $\mathrm{Cl}$ y memoria de trabajo se realizaron de forma individual en un tiempo medio de 90 minutos por 
alumno. Las pruebas de comprensión lectora, atención e inhibición, se realizaron de forma grupal a lo largo de una sesión de 90 minutos por grupo. Por último la cumplimentación del cuestionario de evaluación del funcionamiento ejecutivo conductual, se realizó de forma individual a lo largo del curso por parte de las 7 tutoras. Al final de la evaluación se realizó un informe de cada alumno para los padres donde aparecía información relativa al nivel de comprensión lectora de sus hijos.

\section{Análisis estadísticos}

Los análisis estadísticos se realizaron con el software Statistical Package for the Social Science (SPSS), version 22.0 (SPSS Inc., Chicago, IL USA). Se realizó un análisis multivariante de la varianza (MANOVA) donde se compararon las puntuaciones de sujetos con niveles de comprensión media-baja y media-alta en las variables de funcionamiento ejecutivo.

\section{RESULTADOS}

La Tabla 1 recoge los resultados de los análisis referidos a las diferencias en las variables neuropsicológicas de funcionamiento ejecutivo de sujetos con comprensión media-baja respecto a sus iguales con una comprensión media-alta. El efecto principal de grupo resultó estadísticamente significativo [Wilk s Lambda $(\Lambda)=.911, F(4,128)=3.13, p=.017, \eta^{2} p=.089$ ] . Los ANOVA s de confirmación ofrecieron diferencias significativas en Letras y Números [ $F(1$, $\left.131)=5.37, p=.022, \eta^{2} p=.039\right]$ así como en omisiones $\left[F(1,131)=9.02, p=.003, \eta^{2} p=\right.$ .064]. Sin embargo no alcanzaron significación estadística las tareas de Dígitos $[F(1,131=$ $\left.1.01, p=.303, \eta^{2} p=.008\right]$ y comisiones $\left[F(1,131)=2.62, p=.108, \eta^{2} p=.020\right]$.

Tabla 1.

Diferencias entre los grupos con comprensión lectora media-baja y media-alta: tareas neuropsicológicas del FE

\begin{tabular}{lcccccc}
\cline { 2 - 7 } & \multicolumn{2}{c}{$\begin{array}{c}\text { Media-baja } \\
(\mathrm{N}=63)\end{array}$} & \multicolumn{2}{c}{$\begin{array}{c}\text { Media-alta } \\
(\mathrm{N}=70)\end{array}$} & & \\
\cline { 2 - 7 } & $\mathrm{M}$ & $\mathrm{DT}$ & $\mathrm{M}$ & $\mathrm{DT}$ & $\mathrm{F}(1,131)$ & $\eta^{2} \mathrm{P}$ \\
\hline Letras y números & 17.56 & 2.08 & 18.44 & 2.31 & $5.37 *$ & .039 \\
Dígitos & 16.30 & 2.49 & 16.84 & 3.42 & 1.07 & .008 \\
Omisiones & 10.95 & 10.40 & 6.50 & 6.41 & $9.02 *$ & .064 \\
Comisiones & 1.97 & 2.35 & 1.37 & 1.90 & 2.62 & .020 \\
\hline$* *=p \leq .001 ; *=p<.05$ & & & & & &
\end{tabular}

En la Tabla 2 se presentan los resultados en relación a las diferencias entre el grupo de comprensión media-baja y media-alta para la evaluación conductual del FE. El efecto principal de grupo resultó estadísticamente significativo [Wilk s Lambda $(\Lambda)=.844, F(11,136)=2.28, p=$ $\left..014, \eta^{2} p=.156\right]$. Los ANOVA s de confirmación ofrecieron diferencias significativas en: iniciativa $\left[F(1,146)=18.92, p<.001, \eta^{2} p=.115\right]$, memoria de trabajo $[F(1,146)=17.02, p<$ $\left..001, \eta^{2} p=.104\right]$, planificación/organización $\left[F(1,146)=15.12, p<.001, \eta^{2} p=.094\right]$, organización de materiales $\left[F(1,146)=12,81, p<.001, \eta^{2} p=.081\right]$ y monitoreo $[F(1,146)=9.70$, $\left.p=.002, \eta^{2} p=.062\right]$. Por otro lado no se encontraron diferencias significativas en: inhibición $\left[F(1,146)=.12, p=.728 \eta^{2} p=.001\right]$, cambio $\left[F(1,146)=2.41, p=.123, \eta^{2} p=.016\right]$ y control emocional $\left[F(1,146)=.22, p=.637, \eta^{2} p=.002\right]$. En relación con los índices, se aprecian diferencias significativas en el índice metacognitivo $\left[F(1,146)=17.79, p<.001, \eta^{2} p=.109\right]$ y no en el de regulación comportamental $\left[F(1,146)=.97, p=.326, \eta^{2} p=.007\right]$. 
FUNCIONAMIENTO EJECUTIVO EN ESTUDIANTES CON DIFERENTES NIVELES DE COMPRENSIÓN LECTORA

Tabla 2.

Diferencias entre los grupos con comprensión lectora media-baja y media-alta: evaluación conductual del FE

\begin{tabular}{lcccccc}
\cline { 2 - 7 } & \multicolumn{2}{c}{$\begin{array}{c}\text { Media-baja } \\
(\mathrm{N}=70)\end{array}$} & \multicolumn{2}{c}{$\begin{array}{c}\text { Media-alta } \\
(\mathrm{N}=78)\end{array}$} \\
\cline { 2 - 7 } & $\mathrm{M}$ & $\mathrm{DT}$ & $\mathrm{M}$ & $\mathrm{DT}$ & $\mathrm{F}(1,146)$ & $\eta^{2} \mathrm{P}$ \\
\hline Inhibición & 10.64 & 1.330 & 10.55 & 1.799 & .12 & .001 \\
Cambio & 10.84 & 1.575 & 10.46 & 1.411 & 2.41 & .016 \\
Control emocional & 9.81 & 1.890 & 9.67 & 1.898 & .22 & .002 \\
Iniciativa & 8.83 & 2.719 & 7.37 & 1.106 & $18.92^{* *}$ & .115 \\
Memoria de Trabajo & 12.03 & 3.718 & 10.24 & .840 & $17.02^{* *}$ & .104 \\
Planificación/Organización & 12.04 & 3.605 & 10.38 & 1.035 & $15.12^{* *}$ & .094 \\
Organización de materiales & 8.29 & 2.520 & 7.23 & .623 & $12.81^{* *}$ & .081 \\
Monitoreo & 12.30 & 3.033 & 11.04 & 1.798 & $9.70^{*}$ & .062 \\
Índice de Regulación & 31.30 & 4.027 & 30.62 & 4.391 & .97 & .007 \\
Conductual & & & & & & \\
Índice Metacognitivo & 53.50 & 14.414 & 46.27 & 4.404 & $17.79^{* *}$ & .109 \\
\hline$* *=p<.001 ; *=p<.05$ & & & & & &
\end{tabular}

\section{DISCUSIÓN}

El objetivo de esta investigación consistió en examinar las diferencias en el funcionamiento ejecutivo entre dos grupos de sujetos de último ciclo de Educación Primaria con distintos niveles de comprensión lectora (medio-alto y medio-bajo). Concretamente, se han analizado las FE de memoria de trabajo, inhibición y atención, así como las FE conductuales valoradas por los profesores.

En relación con la memoria de trabajo, los resultados mostraron una ejecución significativamente inferior del grupo con nivel medio-bajo en tareas que requieren memorizar letras y números. Estos resultados están en la línea de investigadores que resaltan la importancia que tiene la memoria de trabajo en la comprensión lectora, tanto en lectores expertos, como en los que presentan dificultades lectoras y en los malos comprendedores (Canet-Juric et al., 2013; Sesma et al., 2009; Swanson y Alexander, 1997). Según Canet-Juric et al. (2013) los sujetos con rendimiento alto en las medidas de memoria operativa tienden a emplear buenas estrategias de comprensión lectora mientras que los estudiantes que obtienen puntuaciones bajas suelen rendir por debajo de la media. No obstante, no se encontraron diferencias en la tarea de dígitos. Esto podría estar relacionado con la una menor complejidad de la misma. Una hipótesis alternativa consistiría en las diferencias en los estímulos utilizados. En este sentido, la tarea de letras y números incluye, además de los dígitos, la manipulación de letras que requiere un procesamiento fonológico adecuado.

En el caso de la atención, también se han encontrado diferencias estadísticamente significativas entre los alumnos que no presentan dificultades de comprensión y los que sí las presentan. En este sentido, son los sujetos con comprensión media-alta los que tuvieron un mayor rendimiento en tareas de atención. Estos datos están en la línea de investigaciones en las que se ha comprobado que los alumnos con dificultades atencionales presentan un menor control sobre la comprensión del texto (Oakhill y Cain, 2007; Mclnnes et al., 2003).

En relación con la inhibición, no aparecen diferencias significativas entre los dos grupos. Este resultado es coincidente con el estudio de Borella et al. (2010) que han encontrado rendimientos similares en el mecanismo de inhibición de distractores y de respuestas de sujtos con niveles diferentes de comprensión lectora. La inhibición parece jugar un papel más significativo en niveles educativos inferiores (Carretti et al., 2009). 
Finalmente, en la evaluación conductual del FE realizada se han encontrado diferencias significativas entre ambos grupos en todos los componentes del índice de metacognición. Esto evidencia mayores problemas de los niños con comprensión media-baja para iniciar, planificar, organizar, gestionar y mantener la información en la memoria de trabajo y monitorear todo el proceso, que los niños sin problemas de comprensión. Estos resultados refuerzan la importancia de los procesos metacognitivos al finalizar la Educación Primaria en la comprensión lectora en la línea de trabajos anteriores (Gioia et al., 2002; Locascio et al., 2010; Pratt, 2000).

Este trabajo no está exento de limitaciones. Las pruebas utilizadas para medir las funciones ejecutivas forman parte de una escala de inteligencia general. Sería interesante evaluar estas variables con pruebas diseñadas específicamente para tal fin. Así mismo, se deberían tener en cuenta otras variables como las características de la familia, el hábito lector del niño, de los padres, o del centro educativo, las prácticas lectoras, etc., ya que son aspectos igualmente fundamentales para entender el desarrollo de la comprensión lectora y sus dificultades.

Por último, de este trabajo se desprenden implicaciones para la práctica psicoeducativa. Sería interesante tener información sobre el funcionamiento ejecutivo desde edades más tempranas, con el fin de potenciarlo. La memoria de trabajo, la atención o la metacognición son factores relevantes que pueden repercutir en la comprensión lectora desde los inicios de su aprendizaje.

\section{CONCLUSIONES}

Se puede concluir que los resultados muestran diferencias estadísticamente significativas entre los niños con diferente nivel de comprensión lectora medio-bajo y medio-alto en las variables de memoria de trabajo y atención. Así mismo, se han encontrado diferencias significativas en la evaluación conductual del funcionamiento ejecutivo realizada por los profesores. Concretamente, es el índice metacognitivo el que evidencia mayores diferencias.

\section{REFERENCIAS BIBLIOGRÁFICAS}

Barth, A. E., Stuebing, K. K., Molfese, P. J., Weiss, B. y Fletcher, J. M. (2009). IQ is not strongly related to response to reading instruction: A meta-analytic interpretation. Exceptional Children, 76(1), 31-51.

Blázquez-Garcés, J. V., Fernández-Andrés, M. I., Sanz-Cervera, P., Tijeras. A., Vélez, X. y PastorCerezuela, G. (2015). Comprensión lectora y oral: relaciones con $\mathrm{Cl}$, género y rendimiento académico de estudiantes de Educación Primaria. International Journal of Developmental and Educational Psychology. Revista INFAD de Psicología, 1(1), 307-314.

Borella, E., Carretti, B. y Pelegrina, S. (2010). La función específica de la inhibición en la comprensión lectora en comprendedores buenas y malas. Diario de Las Discapacidades de Aprendizaje , 43(6), 541-552.

Brock, S. E. y Knapp, P. K. (1996). Reading comprehension abilities of children with attention deficit/hyperactivity disorder. Journal of Attention Disorder, 3, 173-85.

Cain, K. (2006). Individual differences in children's memory and reading comprehension: An investigation of semantic and inhibitory deficits. Memory, 14(5), 553-569.

Cain, K. y Oakhill, J. (2004). Reading comprehension difficulties. In Handbook of children's literacy (pp. 313-338). Springer Netherlands.

Canet-Juric, L., Burin, D., Andrés, M. L. y Urquijo, S. (2013). Perfil cognitivo de niños con rendimientos bajos en comprensión lectora. Anales de Psicología, 29(3), 996- 1005.

Carretti, B., Borella, E., Cornoldi, C. y de Beni, R. (2009). Role of working memory in explaining the performance of individuals with specific reading comprehension difficulties: A meta-analysis. Learning and Individual Differences, 19, 246-251. 


\section{FUNCIONAMIENTO EJECUTIVO EN ESTUDIANTES CON DIFERENTES NIVELES DE COMPRENSIÓN LECTORA}

Cartoceti, R. (2012). Control inhibitorio y comprensión de textos: evidencias de dominio especifico verbal. Revista Neuropsicología Latinoamericana, 4(1), 65-85.

Cuetos, F., Rodríguez, B., Ruano, E., y Arribas, D. (2007). PROLEC-R. Evaluación de los procesos lectores revisada. Madrid: TEA Ediciones.

Filippetti, V. y López, M. B. (2014). The role of executive functions in academic competences: An analytical review (pp. 305-322). En Bennett, K. P. (Ed.). Executive functioning. Role in early learning processes, impairments in neurological disorders and impact of cognitive behavior therapy. New York: Nova Science Publishers, Inc.

Filippetti, V. y López, M. B. (2016). Predictores de la Comprensión Lectora en Niños y Adolescentes: el papel de la Edad, el Sexo y las Funciones Ejecutivas. Cuadernos de Neuropsicología/Panamerican Journal of Neuropsychology, 10(1), 23-44.

García-Madruga, J. A. y Fernández, T. (2008). Memoria operativa, comprensión lectora y razonamiento en la Educación Secundaria. Anuario de Psicología/The UB Journal of Psychology, 39(1), 133-158.

Ghelani, K., Sidhu, R., Jain, U. y Tannock, R. (2004). Reading comprehension and reading related abilities in adolescents with reading disabilities and attention deficit/hyperactivity disorder. Dyslexia, 10, 364-84.

Gioia, G. A., Isquith, P. K., Guy, S. C. y Kenworthy, L. (2000). Behavior Rating Inventory of Executive Function: BRIEF. Odessa, FL: Psychological Assessment Resources.

Gioia, G. A., Isquith, P. K., Kenworthy, L., Barton, R. M. (2002). Profiles of everyday executive function in acquired and developmental disorders. Child Neuropsychology: A Journal on Normal and Abnormal Development in Childhood and Adolescence, 8(2), 121-37.

Gómez Veiga, I., Vila, J. O., García-Madruga. A., Contreras, A. y Elosúa, M. R. (2013). Comprensión lectora y procesos ejecutivos de la memoria operativa. Psicología Educativa, 19( 2), 103-111.

IBM Corp. Released 2013. IBM SPSS Statistics for Windows, Version 22.0. Armonk, NY: IBM Corp.

Kendeou, P., Broek, P., Helder, A. y Karlsson, J. (2014). A cognitive view of reading comprehension: implications for reading difficulties. Learning Disabilities Research and Practice, 29(1), 10-16.

Locascio, G., Mahone, E. M., Eason, S. y Cutting, L. (2010). Executive dysfunction among children with Reading comprehension deficits. Journal of Learning Disabilities, 43(5), 441-454.

López-Escribano, C., De Juan, M. R. E., Gómez-Veiga, I. y García-Madruga, J. A. (2013). A predictive study of reading comprehension in third-grade Spanish students. Psicothema, 25(2), 199205.

McInnes, A., Humphries, T., Hogg-Johnson, S. y Tannock, R. (2003). Listening comprehension and working memory are impaired in attention-deficit hyperactivity disorder irrespective of language impairment. Journal of Abnormal Child Psychology, 31(4), 427-443.

Mezzalira, M. A. y Boruchovitch, E. (2014). Motivación para leer y comprensión lectora de estudiantes brasileños. Educatio Siglo XXI, 32(2), 119-138.

Miranda, A., Fernández, M. I., Robledo, P. y García, R. (2010). Comprensión de textos de estudiantes con trastorno por déficit de atención/hiperactividad: ¿qué papel desempeñan las funciones ejecutivas? Revista de Neurología, 50(3), 135-142.

Nouwens, S., Groen, M. A. y Verhoeven, L. H. (2016). Working memory relates to children's reading comprehension: The importance of domain-specificity in storage and processing. Reading and Writing, 1-16.

Oakhill, J. y Cain, K. (2007). Issues of causality in children's reading comprehension. Reading Comprehension Strategies: Theories, Interventions, and Technologies, 47-72.

Prat, A. (2000). Habilidades cognitivo lingüísticas y tipología textual. En Jaume Jorba, Isabel Gómez 
y Ángeles Prat. Hablar y escribir para aprender. Uso de la lengua ensituación de enseñanzaaprendizaje desde las áreas curriculares. Barcelona. España: Editorial Síntesis.

Seisdedos, N. (2012). d2, Test de atención. Adaptación española (4 ed.rev.). Madrid: TEA Ediciones.

Sesma, H. W., Mahone, E. M., Levine, T., Eason, S. H. y Cutting, L. E. (2009). The contribution of executive skills to reading comprehension. Child Neuropsychology, 15, 232-246.

Solan, H. A., Shelley-Tremblay, J. F., Hansen, P. C. y Larson, S. (2007). Is there a common linkage among reading comprehension, visual attention, and magnocellular processing? Journal of Learning Disabilities, 40(3), 270-278.

Spinillo, A. G. (2013). A dimensão social, linguística e cognitiva da compreensão de textos: considerações teóricas e aplicadas. Compreensão de Textos: Processos e Modelos, 171-198.

Swanson, H. L. y Alexander, J. E. (1997). Cognitive processes as predictors of word recognition and reading comprehension in learning-disabled and skilled readers: Revisiting the specificity hypothesis. Journal of Educational Psychology, 89(1), 128-158.

Wechsler, D. (2010). Escala de Inteligencia de Wechsler para niños- IV: Wisc-IV. Madrid: TEA Ediciones. 
